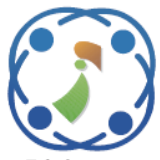

\title{
Human, Robotics Close Motion Classification Based on the Micro Doppler Signature Using HSVD
}

\author{
Mahmood Hamza Al-Mufraji ${ }^{1} \quad$ Thamir R. Saeed $^{1 *} \quad$ Ansam Subhi Jabbar $^{1}$ \\ Jafar W. Abdul Sadah ${ }^{2}$ \\ ${ }^{I}$ Electrical Engineering Department, University of Technology, Iraq. \\ ${ }^{2}$ Communications Engineering Department, University of Baghdad, Iraq. \\ * Corresponding author’s Email: thamir_rashed@yahoo.com
}

\begin{abstract}
In many applications, in industry and laboratory, there is a robot with a human, in close motion, to satisfy the work requirement. Therefore, for safety and monitoring the quality, the classification between them is important. The robot is considered as a semi-rigid object because it has many parts in the half upper, while the lower half is rigid, and a human is a non-rigid object. This paper presents a practical result which investigates the classification between these two objects based on the micro-Doppler signatures. The object's data were acquired using an S-band 2.4 GHz radar. The improved Stockwell transform was used to analyze the radar received signal in the timefrequency domain and feature extraction. The singular value decomposition is used for data filtering based on the Hankel matrix (HSVD) while using the Temporal Radial Basis Function to satisfy the classification. The classification accuracies gained are; $96.4 \%-100 \%$ for semi-rigid, $92 \%-95 \%$ for non-rigid closed objects.
\end{abstract}

Keywords: Micro-doppler, Semi-rigid body, Non-rigid body, Classification, Singular value decomposition, Hankel matrix, Temporal radial basis function.

\section{Introduction}

In most industrial applications and laboratory, there are robots and workers (human) in close motion (co-working), therefore, to satisfy the safety and quality of the required work, and identify the state of the robot [1] and Avoid robot malfunction [2], the motion monitoring and classification is important. Many sensors can satisfy this monitoring; the most famous are the cameras. However, the limitation of the camera is the blind spot, and cannot use it in everywhere and at all time, therefore, can use the radar [3].

In this context, the non-rigid object (Human) have many rigid parts. These rigid parts have different motions. Therefore, when the radar illuminates these objects, there are different frequency components will be produced (modulation) [4]. The main object body produces a Doppler frequency caused by the main movement, while object body parts produce the micro-Doppler
(MD) caused by their motions [5]. Accordingly, the Doppler and micro-Doppler are representing the signatures of the moving objects and include the properties of the transmitted signal (frequency) and the velocity and direction of these moving objects [6-8]. Therefore, the Doppler and micro-Doppler have been used in classifying process for different end applications over the years [9].

In effect, the semi-rigid object (robot) which has the main part (the lower half) as rigid, while it has some parts in motion (in the upper half). In some application, the main part of the semi-rigid object in motion as a single piece (rigid), and in other application is in a fixed situation as shown in Fig. 1.

In general form, the difference between the semi-rigid, and non-rigid is in the nature of the upper half motion and the lower half of the main body. Where, the motion of the semi-rigid (robotic) upper half is periodically, and the lower half is fixed, while in non-rigid (human), it has rotation or 


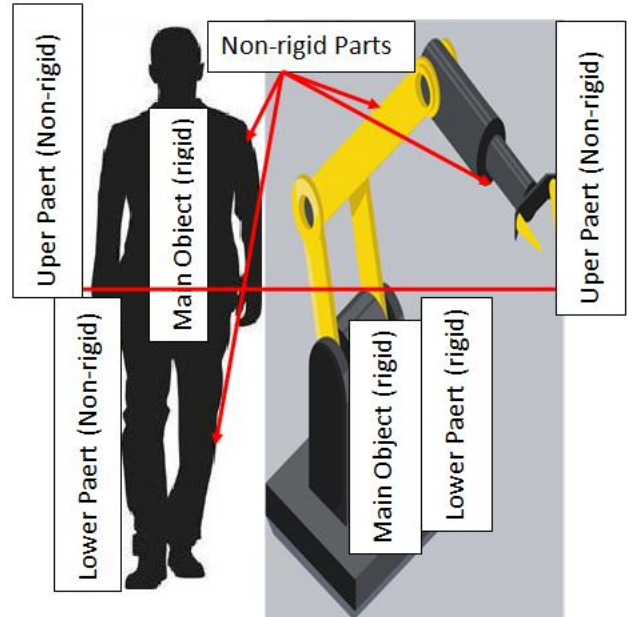

Figure. 1 The difference between Human and robot in motion

vibration in all parts with the fixed motion of the main body, as in Fig. 2.

In this context, When the human and the robot are in an effective state, two different features have been considered: the time-velocity (TV) feature method, and the cadence-velocity (CV) feature method. The method TV feature which can extract the Doppler from it, while the CV tends to the usage the acceleration, in our work will be used the Doppler features [10]. Furthermore, the Short-time Fourier transform (STFT) is used to analyze the received signal and then extracted the required signatures in the time-frequency domain [11].

Several studies have dealt with the analysis of the object's motion by using the Doppler and microDoppler and other features. Some researchers have studied the effect of the transmission media of the radar signal, free space, the wall, and trees [12-15]. While [16, 17] were studying the algorithms of classifications, where, Empirical Mode Decomposition, Support Vector Machines, artificial neural networks, and linear predictive coding have been used. In this context, many other studies have dealt with the types of moving objects and identifying their motion, were $[13,15]$ for vehicle classification, while $[17,18]$ are working to classify the human and animal motions. A human walking mode identification was done $[11,19,20]$ by calculating the velocities of reference points on the human body. A robotic motion identification by using the Inertial Measurement Unit (IMU) signal was presented in [1]. In this context, [1] was gaining $90 \%$ classification, the leak of this work is in using the wavelet in pre-processing, and then uses the wavelet's high and low frequency to determine the starting of the motion, while in this case some information will be lost. As for, the Ref. [11] was

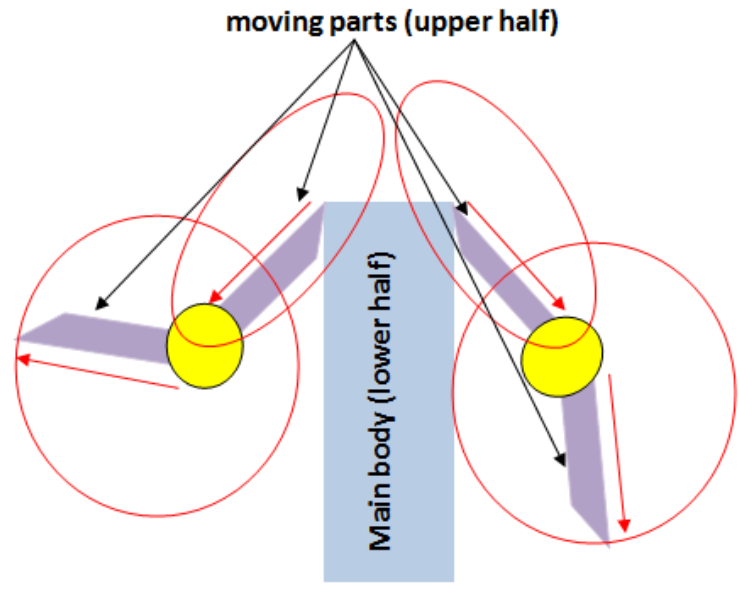

(a)

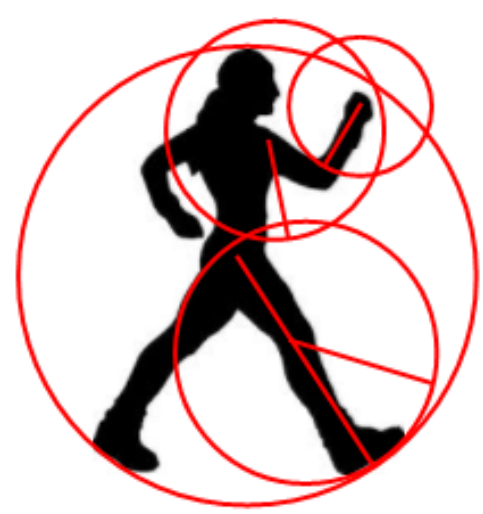

(b)

Figure. 2 The motion of Semi-Rigid and Non-rigid objects: (a) Semi-Rigid body (representation) and (b) non-rigid body (human)

not concerned with the classification rate, while his concentration was on the separation of different object motion. In [21] a human activity recognition by using a convolutional neural network for humanrobotic interaction was presented. The robotic behavior classification was presented in [2], where, multiple robotic behaviors were classified and recognized to avoid the failure in co-working with humans. In the work of ref. [2], The HMM was used as a classification algorithm, which is depending on the state, while he uses the semantic reasoning for representing the human motion, this tends to confuse with HMM states.

Other researchers' works $[4,10]$ were concentrated on the optimality of features, where, in [4] a micro-doppler feature extraction algorithms were presented and applied to a data of moving ground targets. The dimensional reduction was carried out using principal component analysis (PCA) and incorporated into the feature extraction process. Extracted features are classified using a support vector machine (SVM) classifier. The drawback of the work in Ref. [4] is by using the 
feature reduction, where this reduction is active in the case and not in others. In this context, the ref. [10] was gained a classification rate of $92 \%$ because he had only two features (the target base velocity and the main limb cadence frequency), and it was possible to increase the percentage of classification if he increased or chose other features. In other words, the work of Ref. [22] is based on the measurement of the distance between each data point and a certain threshold, where, this give a leak in the classification caused by noisy measurement data.

In effect, in indoor applications, especially some factory and laboratory, there are semi-rigid and nonrigid objects, robotic and human in co-working). Therefore, the accuracy of classification is based on the features of the objects, while these features depend on the signatures of that object. Therefore, the accuracy of signatures extraction is related to the object's motion, sensory properties, and short time Fourier transform (STFT) which is used. The computation of the STFT is to obtain information concerning the variation in the time of the frequency content of the signal [22].

Despite the advances of the previous works, the gap of these studies did not address the semi-rigid, which expresses the robot, which is a co-working with a human in the factories. In all the previous studies, traditional STFT was used, and did not pay attention to its effect on the feature extraction and thus on the classification

In this paper, the improved Stockwell transforms with optimal parameters was used to analyze the Doppler and micro-Doppler signature and then increasing the accuracy of the features extraction. Also, the singular value decomposition (SVD) is used for data filtering based on the Hankel matrix, while the Temporal Radial Basis Function to satisfy the classification was used. The drawbacks which appeared in previous works has been avoided and the average classification rate of $95.65 \%$ has been gained.

The rest of the paper is organized as follows. In section 2, Radar Received Signal and Doppler effect. The Time-Frequency Analysis in section 3. The Denoising stage is presented in section 4, while the Temporal Radial Basis Function is presented in section 4. In section 5, an experimental case study is presented. The simulation and experimental results and discussion of classification are presented in section 6. The conclusions are summarized in section 7 .

\section{Radar received signal and doppler effect}

The frequency of the received signal is [23];

$$
f_{R}=f_{T} \mp f_{D}
$$

Where;

$f_{R}$ - Received frequency.

$f_{T}$ - Transmitted frequency.

$f_{D}$ - Doppler frequency

$$
f_{D}=-f_{T} \frac{2 v}{c}=\frac{1}{2 \pi} \frac{d \varphi(t)}{d t}
$$

$\mp$ - depends on the motion direction of the object (+ toward the transmitter, - away from the transmitter).

$v$ - object velocity.

$c$ - transmitted signal velocity.

$\varphi(t)$ - phase difference between received and transmitted signal.

The received signal frequency which is intercalated the object movement properties [12] according to the object's physical nature (rigid or non-rigid). Therefore, the rigid object produces a shift in transmitting signal frequency, while the nonrigid object produces a band shift in that frequency called micro-Doppler. The Doppler is caused by the motion of the main part of the object (rigid part), while the micro-Doppler is caused by vibration or rotation of some parts of that moving object (nonrigid parts) as shown in Fig. 2.

Then from this Doppler, the object's velocity and direction can be extracted, while from the microDoppler, the velocity of the object's parts is extracted. In effect, the object can be recognized based on these Doppler and micro-Doppler, which are represented as the object's signatures [24]. In this context, the received Doppler (MD) as a function of time which shows the variation of the object (object's parts) with the time, where, the m-D frequency spectrum consists pairs of harmonic spectral lines around the transmitted frequency, as [6];

$$
s(t)=A \sum_{n=-\infty}^{\infty} J_{n}(\beta) e^{\left(j\left(2 \pi f_{T}+n \omega_{v}\right) t\right)}
$$

where;

$A$ - object cross-section from the scattered point. point.

$\omega_{v}$ - radian frequency oscillation of scattered kind.

$J_{n}(\beta)$ - nth-order Bessel function of the first 
In this context, the instantaneous Doppler frequency induced by the scattered points is;

$$
\begin{aligned}
f_{D}=\frac{1}{2 \pi} \frac{d \varphi(t)}{d t} & =\frac{1}{2 \pi} \beta \omega_{v} \cos \left(\omega_{v} t\right) \\
& =\frac{2}{\lambda} D_{v} \omega_{v} \cos \left(\omega_{v} t\right)
\end{aligned}
$$

where;

$\beta=\frac{4 \pi D_{v}}{\lambda}$

$D_{v}$ - amplitude of the scattered point.

$\lambda$ - transmitted wavelength signal.

Then, this effect on the transmitted signal will appear as a modulation in the received signal, as;

$\varphi(t)=\beta \sin \left(\Omega t+\theta_{o}\right)$

where;

$\Omega$ - rotation or vibration rate.

$\theta_{o}$ - rotation or vibration angle at time $\mathrm{t}=0$.

Therefore, the Doppler one rotating or scattering point is;

$$
s(t)=A e^{\left(j\left(2 \pi f_{T} t+\beta \sin \left(\Omega t+\theta_{o}\right)\right)\right)}
$$

then, if there are $\mathrm{N}$ rotating scatters on an object, then caused $\mathrm{N}$ different initial rotating angles as;

$$
\theta_{k}=\theta_{o}+k 2 \pi / N \quad \text { for } k=0, \ldots \ldots . . N-1
$$

then, the total received signal is;

$$
s(t)=\sum_{k=0}^{N-1} A e^{\left(j\left(2 \pi f_{T}+\beta \sin \left(\Omega t+\theta_{o}+k 2 \pi N\right)\right)\right)}
$$

\section{Time-frequency analysis}

The Fourier transform represents a powerful tool to extract the frequency of the signal, while when the frequency is the time variant, this tool is not efficient. Therefore, a Time-Frequency Transforms, which appear the time-dependent frequency information, may be considered powerful tools [25].

Many time-Frequency transforms have been used in previous researches, where that usage is dependent on the required information. Also, the difference between these transforms is the resolution and processing time and the interference cross terms, where each one has advantages on one side and disadvantages on the other.

The Wigner-Ville (WV) transform is a powerful tool which can make a balance between the resolution, process time, and the interference term.
Where this transform can give a high resolution in time-frequency resolution, but it is limited by interference cross term. Therefore, to gain these advantages and to overcome the disadvantages of the time-frequency transform, the Stockwell transform (ST) can be used; which is a hybrid between the short-time Fourier transform (STFT) and wavelet transform (WT). After that, for more accuracy, the improvement of the ST with optimal parameters to control the Gaussian window is used, as [26];

$$
\begin{gathered}
S_{x}^{m, p, k}(\tau, f)= \\
\int_{-\infty}^{+\infty} x(t) \frac{|f|}{\left(m f^{p}+k\right) \sqrt{2 \pi}} e^{\frac{-(t-\tau)^{2} f^{2}}{2\left(m f^{2}+k\right)^{2}}} e^{-i 2 \pi f t} d t
\end{gathered}
$$

where;

$m, k$, and $p-$ Gaussian window control parameters.

It is often beneficial the understanding of the physical phenomena of the object, which is lead to efficient extraction of the required features, and then the classification problem has been solved efficiently also. Therefore, the object features, Signal energy, Average Doppler frequency, Total bandwidth, Doppler offset, Bandwidth without micro-Doppler, Standard deviation (STD) of the lower frequency envelope, and Standard deviation (STD) of upper-frequency envelope $[11,17,19]$, are used for object classification. Where these features are derived from the basic object's parameters like the object's micro-Doppler period, micro-Doppler energy, and range-weighted object energy. The calculating of the micro-Doppler period starts with accumulating the object energy around the bulkscattered and depending on the direction of motion [27] as;

$$
\begin{gathered}
E_{\text {micro-Doppler }}[n]= \\
\sum_{k>k_{\text {bulk }}+k_{o}}\left|S_{x}^{m, p, k}(\tau, f)\right|^{2}
\end{gathered}
$$

where;

$k$-center frequency.

The discrete autocovariance function $\mathrm{C}_{\mathrm{xx}}(\tau)$ provides a measure of the period of a discrete-time sequence $\mathrm{E}_{\text {micro-Doppler }}[\mathrm{i}]$.

$$
\begin{aligned}
& C_{x x}(\tau)=\frac{1}{M} \sum_{i-1}^{M-\tau}\left(E_{\text {micro-Doppler }}[i]-\right. \\
& \left.\hat{\bar{E}}_{\text {micro-Doppler }}[i+\tau]\right)\left(E_{\text {micro-Doppler }}[i+\tau]-\right. \\
& \left.\hat{\bar{E}}_{\text {micro-Doppler }}\right)
\end{aligned}
$$

where; 


$$
\begin{aligned}
& \hat{\bar{E}}_{\text {micro-Doppler }}=\frac{1}{M} \sum_{i=1}^{M} E_{\text {micro-Doppler }}[i] \\
& \tau=0,1,2 \ldots \ldots \ldots . . ., \mathrm{M} \text { ( lag variable }) \\
& M \text { - number of samples. }
\end{aligned}
$$

The micro-Doppler period is the time difference between the peak at zero lag and the next closest peak of $C_{x x}(\tau)$. The micro-Doppler period is helped to know the periodic of the motion.

\section{The denoising stage}

Singular value decomposition (SVD) provides a convenient way of decomposing a matrix, and eliminate the random noise which perhaps contains some data which are interested, by selecting appropriate singular values [28].

The SVD matrix is;

$$
X=U S V^{T}=\left[\begin{array}{lll}
u_{1} & \ldots & u_{n}
\end{array}\right]\left[\begin{array}{ccc}
\sigma_{1} & \cdots & 0 \\
\vdots & \ddots & \sigma_{m} \\
0 & \cdots & 0
\end{array}\right]
$$

where

$U, V$ - left, right singular orthogonal matrices, respectively,

$U-\mathrm{n} \times \mathrm{n}$,

$V-\mathrm{m} \times \mathrm{m}$,

$S$ - a diagonal matrix with a size of $\mathrm{n} \times \mathrm{m}$;

the elements $\sigma_{s}(1 \leq \mathrm{s} \leq \mathrm{m})$ of matrix $S$ are the singular values of matrix $X$; and $\sigma_{1}>\sigma_{2}>\cdots \sigma_{m}>$ 0 .

Then, the data with $m$ traces in each frequency slice of the frequency domain denoted by $a_{t}(1 \leq \mathrm{t} \leq$ $\mathrm{m})$ can be arranged in a Hankel matrix as [28] :

$H=\left[\begin{array}{ccc}a_{1} & a_{2} \ldots & a_{m-p+1} \\ a_{2} & \cdot & \cdot \\ a_{m} & a_{p+1} & a_{m}\end{array}\right]$

\section{The classification stage}

The classification stage has been based on the Temporal Radial Basis Function (TRBF) neural network, as shown in Fig. 3.

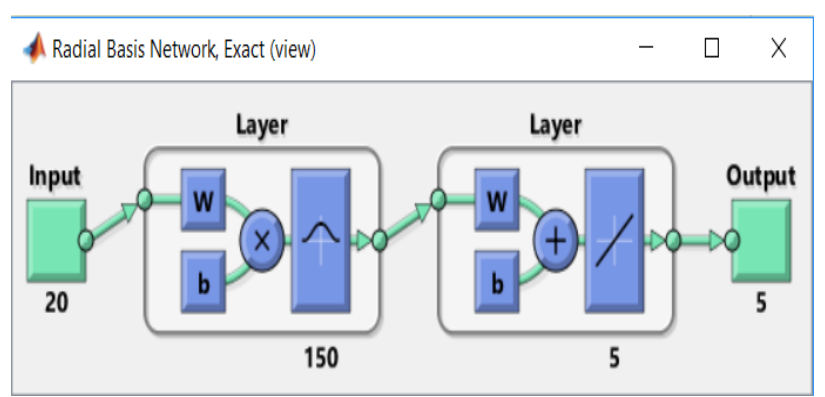

Figure. 3 Temporal radial basis function (TRBF) neural network
This stage is affected by many factors; one of the most powerful ones is the number of training and testing patterns. Therefore, the increasing of the training pattern will cause to undermine the similarity between the patterns to appear the difference between these patterns. The output of the TRBF ' $O$ ' according to the radial basis function of n'. The input to the radbas transfer function is the vector distance between its weight vector ' $\mathrm{w}$ ' and the input vector ' $a$ ' (the elements of Hankel matrix) multiplied by the bias 'b,' where [29];

$$
\begin{aligned}
& n=\|w-a\| b \\
& O=\operatorname{radbas}(n)=\exp \left(-n^{2}\right)
\end{aligned}
$$

\section{Experimental case study}

An Ancortek Inc.[30], is the S-band radar, which has the parameters as in Table 1 . In the research laboratory, many experiments have been done in an indoor environment for objects features, analysis using Stockwell transform with optimal parameters as in Table 2.

Table 1. Radar properties

\begin{tabular}{|l|l|}
\hline Parameters & Values \\
\hline Center Frequency & $2.4 \mathrm{GHz}$ \\
\hline Waveform & FMCW-Saw tooth \\
\hline BW & $400 \mathrm{MHz}$ \\
\hline Sweep Time & $0.5 \mathrm{msec}$. \\
\hline Sampling Number & 128 \\
\hline Polarization & Horizontal \\
\hline
\end{tabular}

Table 2. ST parameters

\begin{tabular}{|c|c|}
\hline Parameters & Values \\
\hline$m$ & 0.45 \\
\hline$p$ & 0.5 \\
\hline$k$ & 0.03 \\
\hline
\end{tabular}

The robotic model was used as in [31] with average error is $5 \mathrm{~mm}$ in the required position for different SNR.

Many measurements have been done; these measurements are; I, Q of the received signals as in Fig. 4, the range against the radial velocity as in Fig. 5 frames against Range as in Fig. 6, and Doppler against the time as in Fig. 7.

The features which can be extracted from these measurements are; signal energy from the received $\mathrm{I}-\mathrm{Q}$, velocity and Doppler range from the velocity against range. In this context, the Doppler statistical features (standard deviations and total bandwidth) are also extracted. 


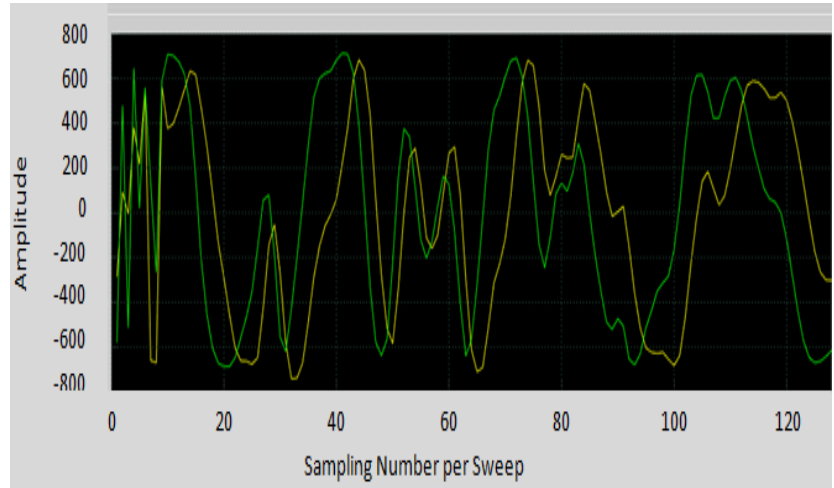

(a)

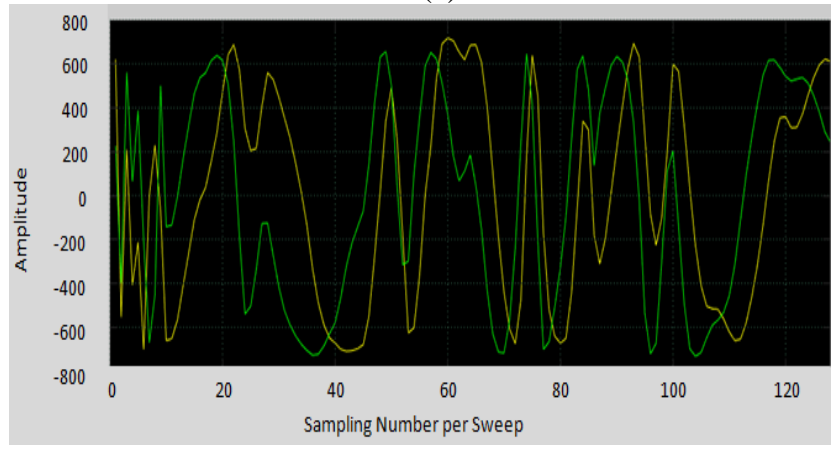

(b)

Figure. 4 I (green), Q (yellow) of reflected signals: (a) semi-rigid body and (b) non-rigid body

Where Fig. 4 indicates the concentration of samples per sweep. This indication referred to the energy related to the motion of the object.

Fig. 5 indicates the elapsed time with a range, in Fig. 5 (a) there is no motion, while in Fig. 5 (b), there is a semi-rigid object in motion, and in Fig. 5 (c) semi-rigid and non-rigid are in motion. From Fig. 5 (b), the periodical motion and time segmentation of semi-rigid object is clear, while in Fig. 5 (c), the periodical motion of semi-rigid and slow motion of non-rigid according to semi-rigid, is clear. Accordingly, the mechanical motion for the semirigid and the non-rigid, it is clear that the semi-rigid is near to square pulse wave, which is related to the periodical motion of semi-rigid (rapid robotic motion). The non-rigid is near to a segment of sinusoidal wave (human motion) slow periodical motion.

Fig. 6 indicates the radial velocity against the range; Fig. 6 (a) is indicated, no motion, Fig. 6 (b) semi-rigid in motion and Fig. 6 (c) semi-rigid and non-rigid in motion. From this figure, the motion period and speed of semi-, and non-rigid objects are clear, where the radial velocity of non-rigid is lower than that of semi-rigid. The semi-rigid (robotic) is faster, and the range of motion is related to the length of its moving parts, while the non-rigid (human) is a slow and limited range of motion.

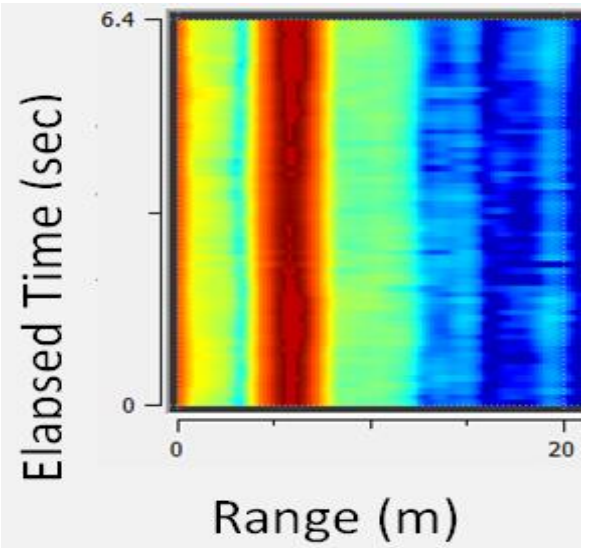

(a)

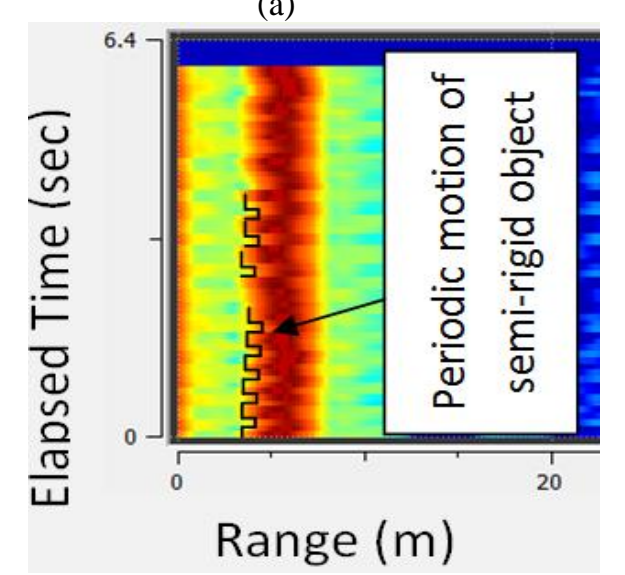

(b)

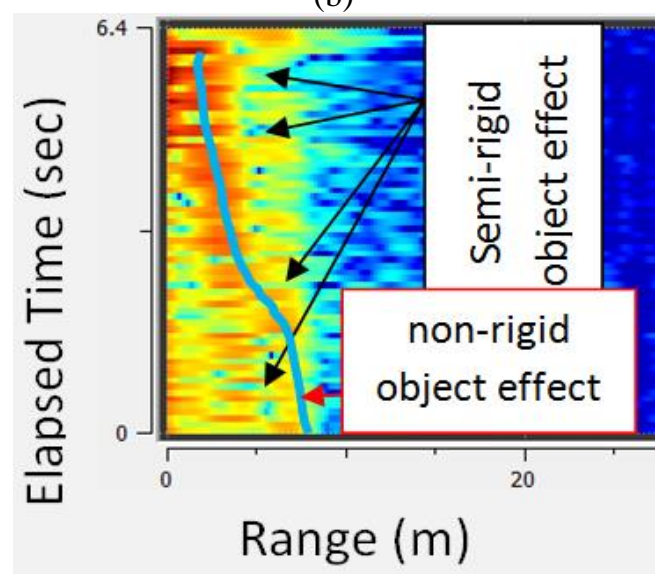

(c)

Figure. 5 Elapsed time (sec.) against a range (m): (a) no motion, (b) semi-rigid object in motion, and (c) semirigid and non-rigid objects in motion

Fig. 7 shows the range frame diagram for the semi-rigid object and non-rigid object in motion. Where this figure appears the variation of signal energy with the range for each frame caused by object motion, it is clear from that figure ((b) and (c)), the expanded of non-rigid motion to the frames more than the expansion of the semi-rigid motion for the same frames, this is because of the fast and slow motion of semi-, and non-rigid objects with frames respectively. 


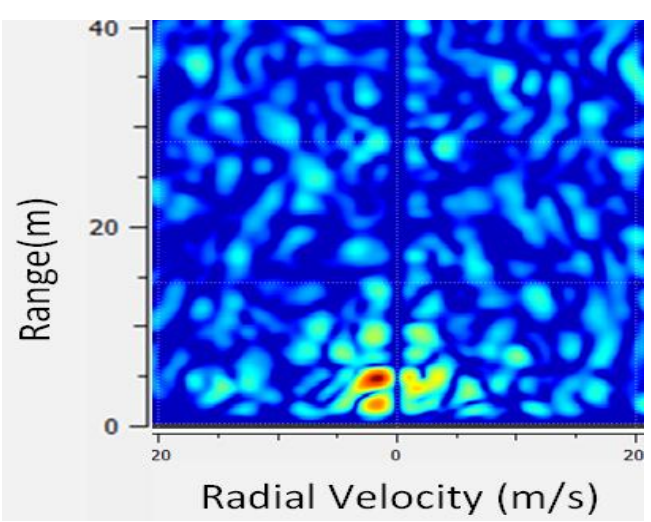

(a)

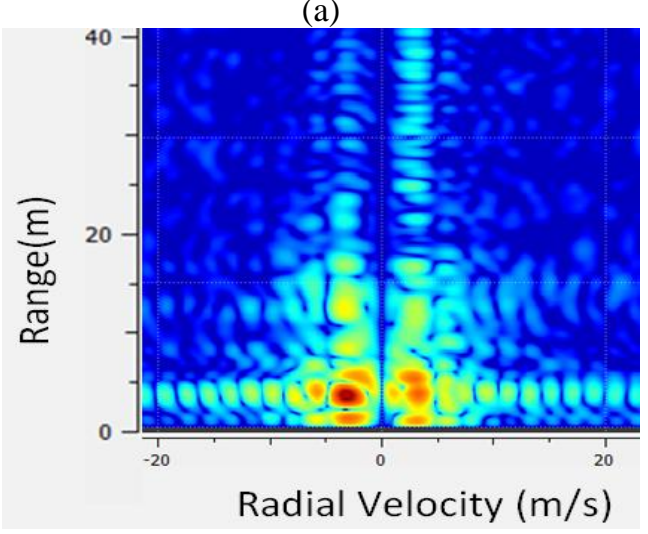

(b)

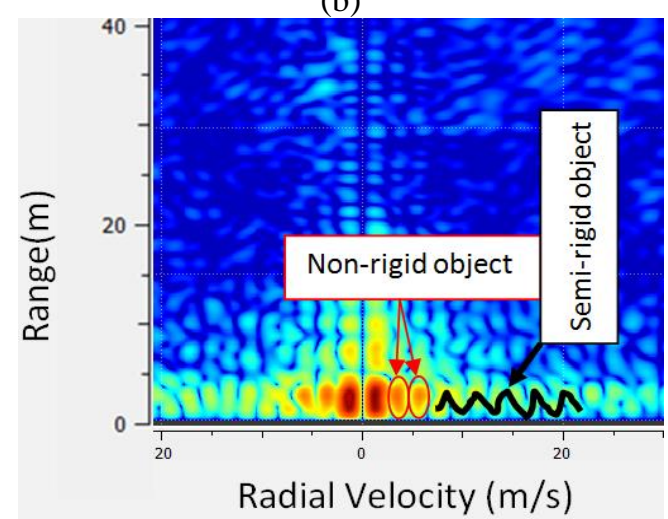

(c)

Figure. 6 Range against radial velocity: (a) no motion, (b) semi-rigid object, and (c) non-rigid and semi-rigid

Then, the extracted features have been arranged in Table 3. for semi-rigid and non-rigid objects.

Table 3. Objects features

\begin{tabular}{|c|c|c|c|c|}
\hline Object & $\begin{array}{c}\text { Energy } \\
\text { (normalized) }\end{array}$ & $\begin{array}{c}\text { Doppler } \\
\text { (average) } \\
\text { Hz }\end{array}$ & $\begin{array}{c}\text { Total } \\
\text { BW Hz }\end{array}$ & $\begin{array}{c}\text { STD } \\
\text { (lower- } \\
\text { upper) } \\
\text { Hz }\end{array}$ \\
\hline $\begin{array}{c}\text { Semi- } \\
\text { Rigid }\end{array}$ & 1 & 75 & 100 & $90-120$ \\
\hline $\begin{array}{c}\text { Non- } \\
\text { rigid } \\
{[32]}\end{array}$ & 0.9794 & 55 & 50 & $32-76$ \\
\hline
\end{tabular}

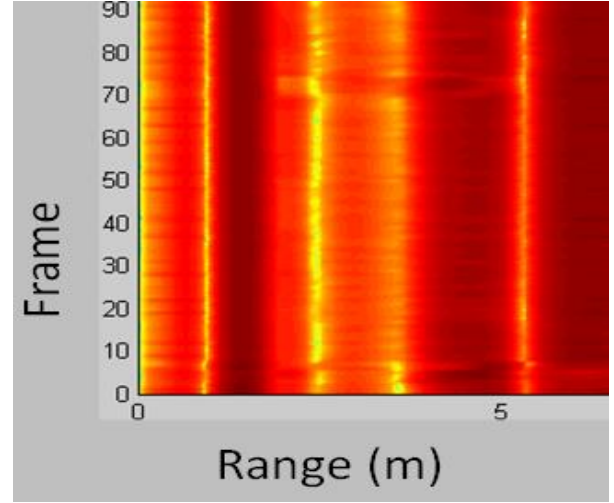

(a)

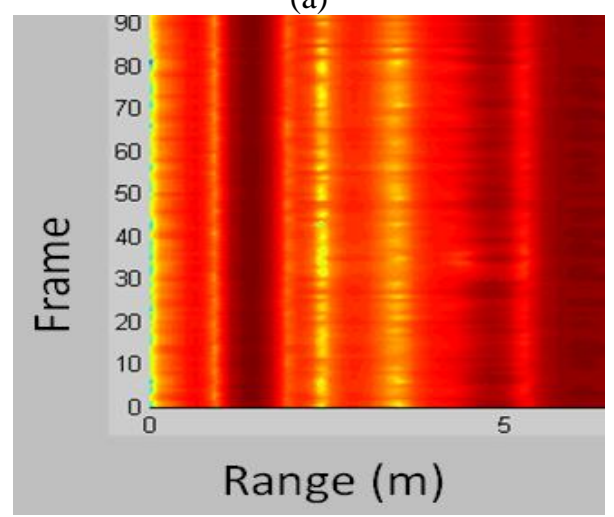

(b)

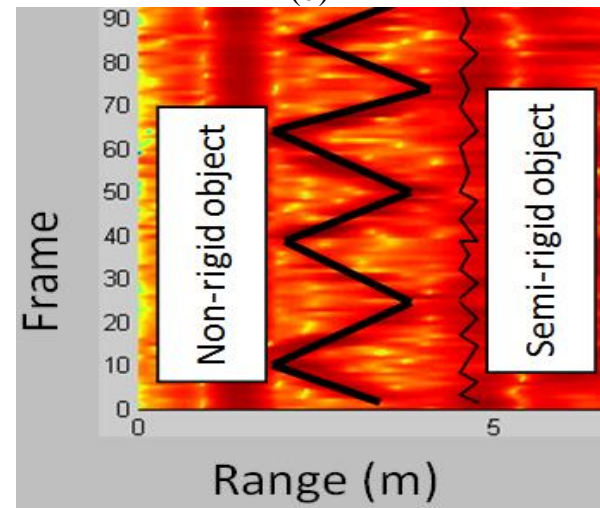

(c)

Figure. 7 Range against the frame: (a) no motion, (b) semi-rigid object, and (c) non-rigid body (human), with a semi-rigid object in motion

\section{Classification results}

The SVD with Hankel matrix (HSVD) approaches have been applied as denoising and arrange the denoising feature, which extracted by STFT. Then TRBF is applied as a classifier with the highest output function assign the class. Then, the Temporal Radial Basis Function was used with the HSVD after the initial testing. The collected data have been split for training and testing, where, 50/10, $50 / 15$, and 50/20 are used. In effect, the classification, resolution depends on the number arrange of features and which the specific feature effects on the classification decision. Then, the 
Table 4. Classification results using the SVM-TRBF classifier

\begin{tabular}{|l|c|c|}
\hline \multirow{2}{*}{ Features used } & \multicolumn{2}{|c|}{ Classification \% } \\
\cline { 2 - 3 } & $\begin{array}{l}\text { Semi-rigid } \\
\text { object }\end{array}$ & $\begin{array}{l}\text { non-rigid } \\
\text { objects }\end{array}$ \\
\hline Energy & $96.4 \%$ & $92 \%$ \\
\hline Doppler +total BW & $97.5 \%$ & $93 \%$ \\
\hline $\begin{array}{l}\text { Doppler +total BW + } \\
\text { STD }\end{array}$ & $100 \%$ & $95 \%$ \\
\hline
\end{tabular}

Table 5. Comparison of present work with the others

\begin{tabular}{|c|l|l|c|}
\hline $\begin{array}{l}\text { Work } \\
\text { [Ref }]\end{array}$ & Features & $\begin{array}{l}\text { Classification } \\
\text { Algorithm }\end{array}$ & $\begin{array}{l}\text { Avg. } \\
\text { Classification\% }\end{array}$ \\
\hline$[1]$ & $\begin{array}{l}\text { IMU } \\
\text { signal }\end{array}$ & $\begin{array}{l}\text { Rule-based } \\
\text { decision logic }\end{array}$ & 90 \\
\hline$[2]$ & $\begin{array}{l}\text { Neural } \\
\text { language }\end{array}$ & HMM $^{2}$ & 83 \\
\hline$[4]$ & $\begin{array}{l}\text { Time- } \\
\text { Frequency }\end{array}$ & SVM $^{3}+$ PCA $^{4}$ & 93.8 \\
\hline$[10]$ & $\begin{array}{l}\text { Energy } \\
\text { +statistical } \\
\text { features }\end{array}$ & $\begin{array}{l}\text { SVM+Liear } \\
\text { Kernal }\end{array}$ & 92 \\
\hline$[11]$ & $\begin{array}{l}\text { Statistical } \\
\text { Features }\end{array}$ & SVM+RBF & 92.005 \\
\hline$[21]$ & $\begin{array}{l}\text { Kinemati } \\
\text { data }\end{array}$ & CNN & 95.71 \\
\hline$[22]$ & $\begin{array}{l}\text { Statistical } \\
\text { Features }\end{array}$ & SVM + PCA & 94.12 \\
\hline $\begin{array}{l}\text { Present } \\
\text { work }\end{array}$ & $\begin{array}{l}\text { Statistical } \\
\text { Features }\end{array}$ & HSVD+TRBF & 95.65 \\
\hline
\end{tabular}

${ }^{1}$ IMU -Inertial Measurement Unit.

${ }^{2}$ HMM -Hidden Markov Model.

${ }^{3}$ SVM -Support Vector Machine.

${ }^{4}$ PCA -Principle Component Analysis.

${ }^{5}$ RBF -Radial Base Function.

classification results as in Table 4 were validated with untrained data for improving the accuracy. The denoising by SVD are made the arrange of the Hankel matrix is efficient, then this tends to classification by TRBF is powerful, that is mean the classification accuracy is overperformed.

These results are overperformed to those reported by $[1,2,4,10,11,22]$. Therefore, for putting our work in a suitable place among the others, a comparison with the other work has been done as in Table 5.

Table 5 shows, there is a significant difference between the present work and the others. From this table, the references $[1,2,4,10,11,22]$ was explaining the lack of their work in section 1 , while the ref [21] is advance our work by $0.06 \%$, and this work depends on the kinematic data. This data is measured by special sensor, and the error in measurement is relatively considered.

The stages of the present work were selected precisely, where, the dancing stage and feature extraction and then arrange it for the classification stage. This work, then, was avoided many drawbacks which appeared in previous works.

Interestingly, our work has been advanced the others; this advancement is related to three points; the time-frequency transforms which was used, specific features which were extracted and used for classification, and the efficient denoising by using SVD, with accuracy in arranging in Hankel matrix with TRBF as a classifier.

The improved Wigner-Ville STFT with optimal parameters as in Table 2 was used, where, this transform was operated as an optimal feature extractor. After that, the TRBF classifier which is used based on the arrange of the features in the Hankel matrix makes the classification process is accurate and easy.

\section{Conclusions}

The present study was designed to determine the importance of the statistical Doppler frequency parameters for the analysis and classification of moving objects (semi, and non-rigid, which is represented robotic and human in co-working). Where, Ancortek Inc. S-band radar has been used for Doppler analysis. Also, the Stockwell transform with optimal parameters has been proved as an overperform tool for analyzing the time frequency of the moving semi-rigid and non-rigid objects according to the reflected signal frequency shift. The object energy and Doppler statistical features can be used in the object classification by using TRBF after denoising by using SVD and arranging in the Hankel matrix to prepare the data for the classification stage. Where, a 96.4-100\% classification rate for semi-rigid objects have been found, while $92-95 \%$ classification rate for non-rigid objects are gained. The radar sensor can be used for safety, quality, and malfunction monitoring in industry crowded locations, where the robot is coworking with a human.

\section{References}

[1] K. Saktaweekulkit and T. Maneewarn, "Motion Classification using IMU for Human-Robot Interaction", In: Proc. of International Conference on Control, Automation and Systems, KINTEX, pp.2295-2299, 2010.

[2] Y. Kobayashi, T. Matsumoto, W. Takano, D. Wollherr, and V. Gabler, "Motion Recognition by Natural Language Including Success and Failure of Tasks for Co-Working Robot with Human", In: Proc. of IEEE International 
Conference on Advanced Intelligent Mechatronics (AIM), pp.10-15, 2017.

[3] T.R. Saeed, S. Mutashar, A. N. Abed, M. Hamza Al-Muifraje, A. Luaibi Shuraiji, and S. Abd-Elghany, "Human motion detection through wall based on Micro-Doppler Using Kalman Filter combined with Convolutional Neural Network", International Journal of Intelligent Engineering and Systems, Vol.12, No.4, pp.317-327, 2019.

[4] A. W. Miller, C. Clemente, A. Robinson, D. Greig, A. M. Kinghorn, and J. J. Soraghan, "Micro-Doppler based object classification using multi-feature integration", In: Proc. of IET Intelligent Signal Processing Conference (ISP 2013), 2013.

[5] M. I. Skolnik, "Introduction to Radar System", Published by McGraw Hill Education (India) Pvt Ltd., 2015.

[6] T. Thayaparan, S. Abrol, E. Riseborough, L. Stankovic, D. Lamothe, and Grant Duff, "Analysis of Radar Micro-Doppler Signatures From Experimental Helicopter and Human Data", IET Radar, Sonar and Navigation, Vol. 1, No. 4, 2007.

[7] S. Björklund, H. Petersson, and G. Hendeby, "Features for micro-Doppler based activity classification", IET Radar, Sonar and Navigation, Vol. 9, No. 9, pp. 1181-1187, 2015.

[8] T. Thayaparan, L. Stankovi'c, and I. Djurovi'c, "Micro-Doppler Based Object Detection and Feature Extraction in Indoor and Outdoor Environments", Journal of The Franklin Institute, Vol. 345, No. 6, 2008.

[9] T. R. Saeed, M. Hamza Al-Muifraje, J. W. Abdul Sadah, and A. N. Abed, "Moving Target Tracking and Classification Based on MicroDoppler Signatures", International Journal of Intelligent Engineering and Systems, Vol.12, No.3, pp. 118-128, 2019.

[10] S. Björklund, H. Petersson, and G. Hendeby, "Features for micro-Doppler based activity classification", IET Radar, Sonar \& Navigation, Special Issue: Micro-Doppler, IET Radar Sonar Navig., Vol. 9, No. 9, pp. 1181-1187, 2015.

[11] M. Zenaldin and R. M. Narayanan, "Radar micro-Doppler based human activity classification for indoor and outdoor environments", In: Proc. SPIE 9829, Radar Sensor Technology, pp.1-10, 2016.

[12] C. Victor Chen, "The Micro-Doppler Effect in Radar", ARTECH HOUSE, 2011.

[13] Y. Li, L. Du, and H. Liu, 'Moving vehicle classification based on micro-Doppler signature", In: Proc. of 2011 IEEE Int. Conf. on Signal Processing, Communications and Computing, pp. 1-4, 2011.

[14] Y. Li, L. Du, and H. Liu, "Noise robust classification of moving vehicles via microDoppler signatures", In: Proc. of the 2013 IEEE Radar Conf., 2013

[15] Y. Li, L. Du, and H. Liu, "Hierarchical classification of moving vehicle based on empirical model decomposition of microDoppler signatures", IEEE Trans. Geosci. Remote Sens., Vol.51, No.5, pp. 3001-3013, 2013.

[16] M. G. Anderson, "Design of multiple frequency continuous wave radar hardware and microDoppler based detection and classification algorithms", PhD dissertation, University of Texas at Austin, 2008.

[17] S. Bjoklund, T. Johansson, and H. Petersson, "Evaluation of a micro-Doppler classification method on mm-wave data", In: Proc. of 2012 IEEE Radar Conf., pp. 934-939, 2012.

[18] G. E. Smith, "Radar object micro-Doppler signature classification", $P h D$ dissertation, Department of Electronic and Electrical Engineering, University College London, 2008

[19] R. Boulic, M. N. Thalmann, and D. Thalmann, "A global human walking model with real-time kinematic personification", The Visual Computer, Vol. 6, No.6, pp. 344-358, 1990.

[20] P. van Dorp and F. C. A. Groen, "Human walking estimation with radar", IET Proc. Radar Sonar Navig., Vol. 150, No. 5, pp. 356365, 2003.

[21] E. Iyiola Olatunji, "Human Activity Recognition for Mobile Robot", IOP Conf. Series: Journal of Physics: Conf. Series 1069, 012148, 2018.

[22] J. Zabalza, C. Clemente, G. Di Caterina, J. Ren, J. J. Soraghan, and S. Marshall, "Robust PCA micro-Doppler classification using SVM on embedded systems", IEEE Transactions on Aerospace and Electronic Systems, Vol. 50, No. 3, 2014

[23] V.C. Chen, F. Li, S.-S. Ho and H. Wechsler, "Analysis of micro-Doppler signatures", IEE Proc.-Radar Sonar Navig., Vol. 150, No. 4, 2003.

[24] T. Wagner, R. Feger, and A. Stelzer, "Radar Signal Processing for Jointly Estimating Tracks and Micro-Doppler Signatures", IEEE Access, Vol. 5, 2017.

[25] P. Setlur, M. Amin, and T. Thayaparan, "Micro-Doppler Signal Estimation for Vibrating and Rotating Objects", IEEE 
Conference, Proceedings of the Eighth International Symposium on Signal Processing and Its Applications, Vol. 2, pp.639-642, 2005.

[26] C. V. Chen and H. Ling, "Time-Frequency Transforms for Radar Imaging and Signal Analysis", Artech House radar library, 2002.

[27] A. Moukadem, D. O. Abdeslam, and A. Dieterlen, "Time-Frequency Domain for Segmentation and Classification of Nonstationary Signals", ISTE Ltd and John Wiley \& Sons, Inc, 2014.

[28] B. Wenda, Y. Zhao, C. An, and S. Hu, "Clutter Elimination and Random-Noise Denoising of GPR Signals Using an SVD Method Based on the Hankel Matrix in the Local Frequency Domain", Sensors, Vol. 18, No. 3422, 2018.

[29] T. R. Saeed, J. Salman, and A. Hussein Ali, "Classification improvement of spoken arabic language based on radial basis function", International Journal of Electrical and Computer Engineering, Vol. 9, No. 1, pp. 402408, 2019.

[30] ANCORTEK INC, 2016, at www.ancortek.com.

[31] J. Salman, A. H. Ali, and T. R. Saeed, "Performance Evaluation of a Forward Kinematic Hand Model", Journal of University of Babylon, Engineering Sciences, Vol.26, No.1, pp.35-41, 2018.

[32] T. R. Saeed, M. Hamza Al-Muifraje, and G. M. Hatem, "Through the Wall, Recognize Moving Targets Based on Micro-Doppler Signatures", International Journal of Electrical and Computer Engineering, Vol. 8, No. 6, pp. 52275237, 2018. 